\title{
Topological superfluid of spinless Fermi gases in p-band honeycomb optical lattices with on-site rotation
}

\author{
Beibing Huang \\ Department of Experiment Teaching, Yancheng Institute of Technology, Yancheng, 224051, China \\ Xiaosen Yang and ShaoLong Wan \\ Institute for Theoretical Physics and Department of Modern Physics \\ University of Science and Technology of China, Hefei, 230026, China
}

October 29, 2018

\begin{abstract}
In this paper, we put forward to another route realizing topological superfluid (TS). In contrast to conventional method, spin-orbit coupling and external magnetic field are not requisite. Introducing an experimentally feasible technique called on-site rotation (OSR) into p-band honeycomb optical lattices for spinless Fermi gases and considering CDW and pairing on the same footing, we investigate the effects of OSR on superfluidity. The results suggest that when OSR is beyond a critical value, where CDW vanishes, the system transits from a normal superfluid (NS) with zero TKNN number to TS labeled by a non-zero TKNN number. In addition, phase transitions between different TS are also possible.
\end{abstract}

PACS number(s): 67.85.Lm, 03.65.Vf, 74.20.-z

\section{Introduction}

Topological superfluid (or superconductor) (TS) has a full pairing gap in the bulk and is labeled by a non-zero integer topological invariant [1, 2]. From the famous bulk-boundary correspondence such a topological integer ensures the existence of gapless excitations on the boundary of the system, in other words Majorana fermions (MF) [3] in vortex core of pairing order parameter. Roughly, MFs are neither fermions nor bosons but non-Abelian anyons 
[4] and play an important role for the realization of fault-tolerant topological quantum computation (TQC) [5]. The application prospect of MFs makes TS become one of the hottest frontiers.

In the condensed matter physics some practical two-dimensional systems have been theoretically proposed to realize TS [6, 7, 8, 9, 10, 11, 12, 13. In terms of these systems the entrance into TS requires subtle adjustment of Hamiltonian and it is very difficult in condensed matter physics, although MFs have been detected in InSb nanowires contacted with one normal $(\mathrm{Au})$ and one superconducting electrode (NbTiN) [14. In the light of the disadvantage for condensed matter, TS has been also suggested in cold Fermi gases owing to their many controllable advantages and operabilities. Following the successful observation p-wave Feshbach resonance (FR), Gurarie et al. [15] show that degenerate Fermi gases near a p-wave FR naturally give a concrete realization of TS. Zhang et al. [16] propose to create TS directly from an s-wave interaction making use of an artificially generated spin-orbit coupling (SOC). In fact, SOC have been realized in a neutral atomic Bose-Einstein condensate (BEC) by dressing two atomic spin states with a pair of lasers and the same technique is also feasible for cold Fermi gases [17, 18]. Realizing that in a dual transformation SOC is formally equivalent to a p-wave superfluid gap, Sato et al. [19] suggest to artificially generate the vortices of SOC by using lasers carrying orbital angular momentum. In terms of the latter two ways, SOC and a large magnetic field are crucial in order to enter into TS.

In this paper we suggest to create TS from spinless Fermi gases in p-band honeycomb optical lattices with so-called on-site rotation (OSR), that rotates every lattice site around its own center but keeps the whole lattice intact and has been realized for triangular optical lattices [20]. As a matter of fact p-band Fermi gases in honeycomb optical lattices in absence of OSR have shown many interesting characteristics, such as ferromagnetism [21] and Wigner crystallization [22, 23] associating with flat bands, f-wave superfluidity with conventional pairing interaction [24]. The motivation for this paper comes from the Wu's work on quantum anomalous Hall effect in the same system [25]. Under single particle picture, Wu found that an arbitrary non-zero OSR not only breaks time-reversal symmetry and changes the topological properties of the system, but also drives a topological phase transition when OSR is beyond a critical value. Here we add on-site attraction interaction between p-band Fermi atoms into Hamiltonian and ask a question whether OSR can drive a phase transition into TS. The results are positive and OSR brings phase transitions not only from normal superfluid (NS) to TS, but also among different TS. From another perspective our work also can be considered as an extension to [24], where f-wave superfluidity without OSR is discussed. Thus we also investigate the effects of OSR on f-wave superfluidity.

Experimentally the route to realize TS suggested here is also feasible. On the one hand by placing two electro-optic modulators at two of three laser beams which coherently superpose to form a honeycomb lattice, OSR is available as illustrated in [26]. On the other hand due to Pauli exclusion principle the occupation of p-band is very convenient as long as the lowest s-band is fulfilled. In addition, on-site attraction interaction can be enhanced by using atoms with large magnetic moments, such as ${ }^{167} \mathrm{Er}$ with $m=7 \mu_{B}$ on which laser cooling has been performed [27]. In contrast to [16, 19], where a pair of extra lasers and a large magnetic field are needed to produce an effective SOC and split two SOC bands respectively, our system is much simpler. 
The organization of this paper is as follows. In section 2 , we give the model and at the mean-field level investigate the ground state of the system by numerically minimizing the thermodynamic potential. In section 3 by calculating TKNN number $I_{T K N N}$ [28] of occupied bands addressing the topological properties of the model, the topological phase diagram is obtained. In addition we also investigate the properties of edge states to prove our results. A brief conclusion is given in section 4 .

\section{Model and Mean-Field Ground State}

The honeycomb optical lattice was realized experimentally by using three laser beams with co-planar propagating wavevectors quite some time age 29]. It is well known that a honeycomb lattice is not a Bravais lattice and there are two inequivalent sites in a unit cell, denoted by $\mathrm{A}$ and $\mathrm{B}$ respectively. Fulfilling the lowest s-band and defining three unit vectors $\vec{e}_{1}=\frac{\sqrt{3}}{2} \vec{e}_{x}+\frac{1}{2} \vec{e}_{y}, \vec{e}_{2}=-\frac{\sqrt{3}}{2} \vec{e}_{x}+\frac{1}{2} \vec{e}_{y}$ and $\vec{e}_{3}=-\vec{e}_{y}$, the Hamiltonian of p-band honeycomb optical lattices with OSR is

$$
H=t_{\|} \sum_{\vec{r} \in A, i}\left[p_{\vec{r}, i}^{\dagger} p_{\vec{r}+\vec{e}_{i}, i}+H . C .\right]-U \sum_{\vec{r} \in A \oplus B} p_{\vec{r} x}^{\dagger} p_{\vec{r} y}^{\dagger} p_{\vec{r} y} p_{\vec{r} x}-\Omega \sum_{\vec{r} \in A \oplus B} \hat{l}_{\vec{r}, z}-\mu \sum_{\vec{r} \in A \oplus B} \hat{n}_{\vec{r}}
$$

where $p_{\vec{r}, i}=\left(p_{\vec{r}, x} \vec{e}_{x}+p_{\vec{r}, y} \vec{e}_{y}\right) \cdot \vec{e}_{i}$ and $p_{\vec{r}, x}\left(p_{\vec{r}, y}\right)$ is the annihilation operator for $p_{x}\left(p_{y}\right)$

band at the lattice site $\vec{r} . \hat{n}_{\vec{r}}=p_{\vec{r}, x}^{\dagger} p_{\vec{r}, x}+p_{\vec{r}, y}^{\dagger} p_{\vec{r}, y}$ and $\hat{l}_{\vec{r}, z}=-i\left(p_{\vec{r}, x}^{\dagger} p_{\vec{r}, y}-p_{\vec{r}, y}^{\dagger} p_{\vec{r}, x}\right)$ represent particle number and orbital angular moment operators. $t_{\|}$is the nearest-neighbor hopping matrix element of atoms in $\sigma$ bonds and positive due to the odd parity of the p-orbital. $U$ $(>0), \Omega(>0)$ and $\mu$ are the on-site interaction strength, on-site rotation angular velocity and chemical potential, respectively. Note that we have neglected the nearest-neighbor atom hopping of $\pi$ bonds and supposed the nearest neighbor distance in the lattice to be unit.

When $U=0$, introducing operator $\phi(k)=\left[p_{A x}(k), p_{A y}(k), p_{B x}(k), p_{B y}(k)\right]^{T}$ and making a unitary transformation $\phi_{n}(k)=U_{n m}(k) \Psi_{m}(k)$, Hamiltonian can be diagonalized exactly. Meanwhile four energy bands can be obtained. Wu found two of four bands always are topological for any nonzero OSR and the others can be topological only if OSR is beyond a critical value [25]. On the basis of this findings, Wu proposed an orbital analogue of the quantum anomalous Hall effect, arising from orbital angular momentum polarization due to OSR. With $\Omega=0$, Lee et al. discussed f-wave superfluidity and charge density wave (CDW) in this system at the mean-field level [24]. Their results show that away from the half filling the system is f-wave superfluidity, while around the half filling superfluidity and CDW coexist and the system is a supersolid. Although superfluidity exists all the time, it is not topological as stated below.

Following the same spirit in [24] we decouple interaction term into CDW channel

$$
H_{\text {int }}^{C D W}=\sum_{\tau=x, y}\left[\sum_{\vec{r} \in A}\left(-\frac{n}{2} U-\frac{\Delta_{C D W}}{2}\right) p_{\vec{r}, \tau}^{\dagger} p_{\vec{r}, \tau}+\sum_{\vec{r} \in B}\left(-\frac{n}{2} U+\frac{\Delta_{C D W}}{2}\right) p_{\vec{r}, \tau}^{\dagger} p_{\vec{r}, \tau}\right]
$$

and pairing channel

$$
H_{\text {int }}^{\text {pairing }}=-\sum_{k}\left[\Delta_{A} p_{A x}^{\dagger}(k) p_{A y}^{\dagger}(-k)+\Delta_{B} p_{B x}^{\dagger}(k) p_{B y}^{\dagger}(-k)+H . C .\right]
$$




$$
=-\sum_{k^{\prime}}\left[\Delta_{n m}\left(k^{\prime}\right) \Psi_{n}^{\dagger}\left(k^{\prime}\right) \Psi_{m}^{\dagger}\left(-k^{\prime}\right)+H . C .\right]
$$

where $n=<\hat{n}_{\vec{r}_{A}}+\hat{n}_{\vec{r}_{B}}>/ 2$ is filling factor of every site, $\Delta_{C D W}=U<\hat{n}_{\vec{r}_{A}}-\hat{n}_{\vec{r}_{B}}>/ 2$, $\Delta_{A}=U \sum_{k}<p_{A y}(-k) p_{A x}(k)>, \Delta_{B}=U \sum_{k}<p_{B y}(-k) p_{B x}(k)>$ are order parameters for CDW and superfluidity. In (3) we also express the pairing channel using quasiparticle $\Psi(k)$. In this representation

$$
\begin{aligned}
\Delta_{n m}(k) & =\Delta_{A}\left[U_{1 n}^{*}(k) U_{2 m}^{*}(-k)-U_{2 n}^{*}(k) U_{1 m}^{*}(-k)\right] \\
& +\Delta_{B}\left[U_{3 n}^{*}(k) U_{4 m}^{*}(-k)-U_{4 n}^{*}(k) U_{3 m}^{*}(-k)\right] .
\end{aligned}
$$

After the mean-field approximation, the Hamiltonian (11) becomes a BdG Hamiltonian $H=\left[\phi^{\dagger}(k), \phi(-k)\right] H_{k}\left[\phi(k), \phi^{\dagger}(-k)\right]^{T}$ and the properties of system are completely decided by the $8 \times 8$ matrix $H_{k}$. Diagonalizing $H_{k}$, we attain spectrum $\epsilon_{i}(k)$ and correspondingly eigenvectors $\varphi_{i}(k)(i=1,2, \cdots, 8)$. Due to particle-hole symmetry inherent in this BdG Hamiltonian, the spectrum are symmetric about zero energy and we assume $\epsilon_{1}(k)=-\epsilon_{8}(k)>0, \epsilon_{2}(k)=-\epsilon_{7}(k)>0, \epsilon_{3}(k)=-\epsilon_{6}(k)>0, \epsilon_{4}(k)=-\epsilon_{5}(k)>0$. Then the thermodynamical potential at zero temperature is

$$
F=\frac{1}{2} \sum_{k}\left[-4 \mu-\epsilon_{1}(k)-\epsilon_{2}(k)-\epsilon_{3}(k)-\epsilon_{4}(k)\right]+\frac{N}{U}\left|\Delta_{A}\right|^{2}+\frac{N}{U}\left|\Delta_{B}\right|^{2}+\frac{N}{2 U} \Delta_{C D W}^{2},
$$

where $N$ is the number of the unit cell. Below we numerically minimize thermodynamic potential $F$ about $\Delta_{A}, \Delta_{B}$ and $\Delta_{C D W}$ for fixed interaction strength $U$. Without loss of generality we choose $\Delta_{A}$ to be real, $\Delta_{B}=\left|\Delta_{B}\right| e^{i \theta}$ and $U / t_{\|}=3.0$.

Fig.1 shows the solutions of the Hamiltonian (11) at the mean-field level for changing chemical potential $\mu$ and OSR $\Omega$. Due to the particle-hole symmetry we only concentrate on negative chemical potential. Fig.1(a) describes the variation of $\Delta_{C D W}$. For $\Omega=0$ CDW is robust, but when $\Omega$ is beyond a critical value $\Omega_{c}$ it vanishes suddenly. This is due to the fact that the appearance of OSR changes the band structures of single particle and breaks the nesting condition for CDW. Numerically we find $\Omega_{c} / t_{\|} \approx 0.4 \sim 0.6$ and is monotonically increasing as the function of chemical potential. Fig.1(b) shows the effect of OSR on particle density and further exemplifies that the variations of band structures driven by OSR cause nonmonotonic behavior of particle density. In contrast, superfluid order parameters $\Delta_{A}, \Delta_{B}$ are more interesting and shown in (c) and (d). On the one hand for $\Omega>\Omega_{c}, \Delta_{A}=\left|\Delta_{B}\right|$ and with the increase of OSR superfluid order smoothly decreases until disappearance. This suppression mechanism of superfluidity consists in time-reversal symmetry broken caused by OSR. While on the other hand for $\Omega<\Omega_{c} \Delta_{A}$ is still decreasing but $\Delta_{B}$ is increasing with $\Omega$. In fact the increase of $\Delta_{B}$ originates from the redistribution of particle density between sites $\mathrm{A}$ and $\mathrm{B}$, in other words the decrease of $\Delta_{C D W}$ as seen in (a). Thus at the mean-field level our calculation suggests that (1) OSR weakens stabilities of CDW and superfluidity and (2) for $\Omega<\Omega_{c}$, superfluidity and CDW coexist and the system is a supersolid.

The optimization of $\theta$ leads to $\theta=\pi$ for all parameters we choose. Below we discuss the effects of OSR on pairing symmetry for $\Omega>\Omega_{c}$. From [24] without OSR and away from the half filling the intraband pairings in (44) have f-wave symmetry with three nodal lines of $k_{x}=0, k_{y}= \pm k_{x} / \sqrt{3}$ and $\pi / 3$ rotation symmetry [Fig.2(d)]. On introducing OSR, 
in terms of the pairing magnitude, nodal lines degenerate into some disconnected regions where intraband gap disappears, and $\pi / 3$ rotation symmetry retains. However real and imaginary parts of pairing break $\pi / 3$ into $\pi$ rotation symmetry. In Fig.2(a) (b) and (c) as an example we show the magnitude, real and imaginary parts of $\Delta_{11}$.

\section{Topological Phase Diagram and Majorana Fermion Modes}

In this section we discuss topological properties of Hamiltonian (1). In terms of our system, it explicitly breaks the time-reversal symmetry due to OSR. Thus TKNN number $I_{T K N N}$ plays a central role in deciding topological nature of the system [28]. TKNN number is defined, by eigenvectors $\varphi_{i}(k)(i=5,6,7,8)$ corresponding to negative energy spectrm of the matrix $H_{k}$, into $I_{T K N N}=\frac{1}{2 \pi i} \int d^{2} k \operatorname{Tr} d A$, where $A$ is a matrix one-form $A_{i j}=A_{i j}^{\nu}(k) d k_{\nu}$

with $A_{i j}^{\nu}(k)=\varphi_{i}^{\dagger}(k) \nabla_{k_{\nu}} \varphi_{j}(k)$. By numerically calculating TKNN number [30], we show the topological phase diagram of the system in Fig.3. For parameter region we choose, there are four different subregions labeled by $I_{T K N N}=1,0,-1,2$ respectively. Moreover by comparison with Fig.1(a) it is easily found that the boundary between $I_{T K N N}=0$ and other TKNN numbers in the direction of $\Omega$ coincides with that of CDW disappearance. This finding is very important and ensures that topological order of our system is not topological CDW [31]. According to the criteria for TS [31] $I_{T K N N}=2$ corresponds to Abelian TS while $I_{T K N N}=1,-1$ are non-Abelian TS. Thus Fig.3 tells us that OSR drives topological phase transition not only from NS to TS, but also between different TS. Here we mention a fact the energy gap of the bulk spectrum closes when topological phase transitions between topologically distinct phases occur.

From the bulk-edge correspondence, a non-trivial bulk topological number implies the existence of gapless edge states localized on open edges of the system. Cold Fermi gases with sharp edges may be realized along the lines proposed in [32. In order to understand the relation between $I_{T K N N}$ and the number of edge states, we study the Hamiltonian (11) with the open boundary condition along the zigzag edge of the honeycomb lattice. The resulting excitation spectrum are depicted in Fig.4 for representative parameter choices. Very explicitly the number of gapless states for every edge is one-to-one correspondence with the TKNN number. For $I_{T K N N}= \pm 1\left(I_{T K N N}=2\right)$ there are one (two) pair(s) of gapless states, while for $I_{T K N N}=0$, gapless state does not exist. Due to particle-hole symmetry, in terms of gapless states, they are Majorana fermion modes. It should also be remembered that the core of a vortex is topologically equivalent to an edge which has been closed on itself. The edge modes we describe are therefore equivalent to the Majorana fermions known to exist in the core of vortices of p-wave superfluids [33].

\section{Conclusions}

In conclusion at the mean-field level we have investigated the effects of OSR on CDW and superfluidity for p-band spinless Fermi gases in honeycomb optical lattices. We found that OSR weakens the stabilities of CDW and superfluidity simultaneously, although superfluidity can survives a larger OSR. This conclusion leads to another important result that once CDW drops out the system enters into topological superfluidity. By numerically calculating 
the TKNN number we obtained topological phase diagram of the system. In addition edge states, i.e. bulk-boundary correspondence are also investigated.

\section{Acknowledgement}

The work was supported by National Natural Science Foundation of China under Grant No. 10675108 and Foundation of Yancheng Institute of Technology under Grant No. XKR2010007.

\section{References}

[1] M. Z. Hasan and C. L. Kane, Rev. Mod. Phys. 82, 3045 (2010).

[2] X.-L. Qi and S.-C. Zhang, Rev. Mod. Phys. 83, 1057 (2011).

[3] E. Majorana, Nuovo Cimento 5, 171 (1937).

[4] D. A. Ivanov, Phys. Rev. Lett. 86, 268 (2001).

[5] C. Nayak, S. H. Simon, A. Stern, M. Freedman and S. Das Sarma, Rev. Mod. Phys. 80, 1083 (2008).

[6] N. Read and D. Green, Phys. Rev. B 61, 10267 (2000).

[7] Y. Tsutsumi, T. Kawakami, T. Mizushima, M. Ichioka and K. Machida, Phys. Rev. Lett. 101, 135302 (2008).

[8] M. Sato and S. Fujimoto, Phys. Rev. B 79, 094504 (2009).

[9] P. A. Lee, arXiv:0907.2681.

[10] L. Fu and C. L. Kane, Phys. Rev. Lett. 100, 096407 (2008).

[11] J. Linder, Y. Tanaka, T. Yokoyama, A. Sudbo and N. Nagaosa, Phys. Rev. Lett. 104, 067001 (2010).

[12] J. D. Sau, R. M. Lutchyn, S. Tewari, and S. Das Sarma, Phys. Rev. Lett. 104, 040502 (2010).

[13] J. Alicea, Phys. Rev. B 81, 125318 (2010).

[14] V. Mourik, K. Zuo, S. M. Frolov, S. R. Plissard, E. P. A. M. Bakkers and L. P. Kouwenhoven, Science 336, 1003 (2012).

[15] V. Gurarie, L. Radzihovsky and A. V. Andreev, Phys. Rev. Lett. 94, 230403 (2005).

[16] C. Zhang, S. Tewari, R.M. Lutchyn and S. Das Sarma, Phys. Rev. Lett. 101, 160401 (2008). 
[17] P. Wang, Z. Yu, Z. Fu, J. Miao, L. Huang, S. Chai, H. Zhai and J. Zhang, arXiv:1204.1887.

[18] L. W. Cheuk, A. T. Sommer, Z. Hadzibabic, T. Yefsah, W. S. Bakr and M. W. Zwierlein, arXiv:1205.3483.

[19] M. Sato, Y. Takahashi and S. Fujimoto, Phys. Rev. Lett. 103, 020401 (2009).

[20] N. Gemelke, E. Sarajlic, S. Chu, arXiv:1007.2677.

[21] S. Zhang, H. Hung and C. Wu, Phys. Rev. A 82, 053618 (2010).

[22] C. Wu, D. Bergman, L. Balents and S. Das Sarma, Phys. Rev. Lett. 99, 070401 (2007).

[23] C. Wu and S. Das Sarma, Phys. Rev. B 77, 235107 (2008).

[24] W. Lee, C. Wu and S. Das Sarma, Phys. Rev. A 82, 053611 (2010).

[25] C. Wu, Phys. Rev. Lett. 101, 186807 (2008).

[26] M. Zhang, H. Hung, C. Zhang and C. Wu, Phys. Rev. A 83, 023615 (2011).

[27] J. J. McClelland and J. L. Hanssen, Phys. Rev. Lett. 96, 143005 (2006).

[28] D. J. Thouless, M. Kohmoto, M. P. Nightingale and M. Nijs, Phys. Rev. Lett. 49, 405 (1982).

[29] G. Grynberg, B. Lounis, P. Verkerk, J.-Y. Courtois and C. Salomon, Phys. Rev. Lett. 70, 2249 (1993).

[30] T. Fukui, Y. Hatsugai and H. Suzuki, J. Phys. Soc. Jpn. 74, 1674 (2005).

[31] M. Sato, Y. Takahashi and S. Fujimoto, Phys. Rev. B 82, 134521 (2010).

[32] N. Goldman, I. Satija, P. Nikolic, A. Bermudez, M. A. Martin-Delgado, M. Lewenstein and I. B. Spielman, Phys. Rev. Lett. 105, 255302 (2010).

[33] A. Kubasiak, P. Massignan and M. Lewenstein, Europhys. Lett. 92, 46004 (2010). 

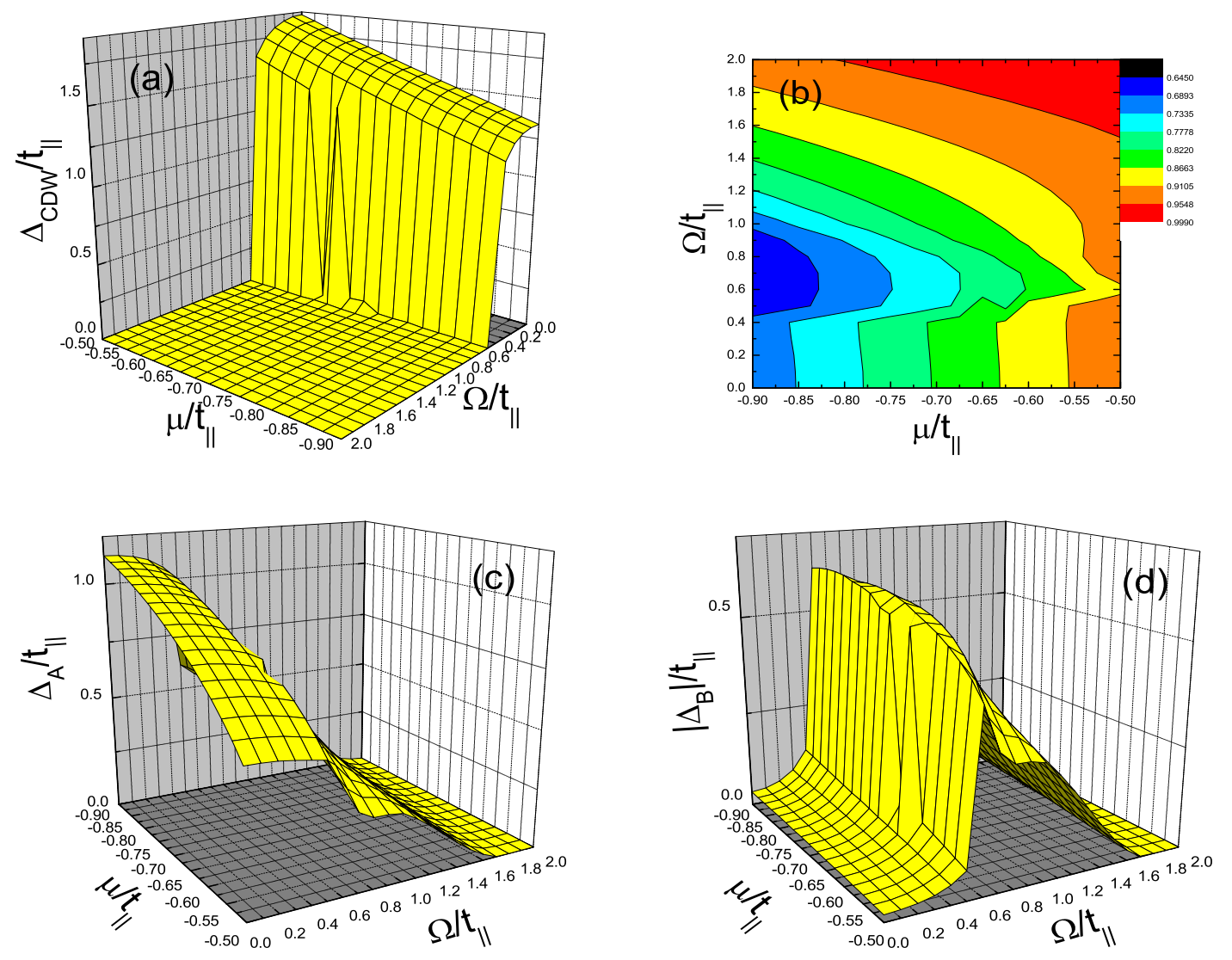

Figure 1: The mean-field solution of the Hamiltonian (1). Parameter $U / t_{\|}=3.0$. 

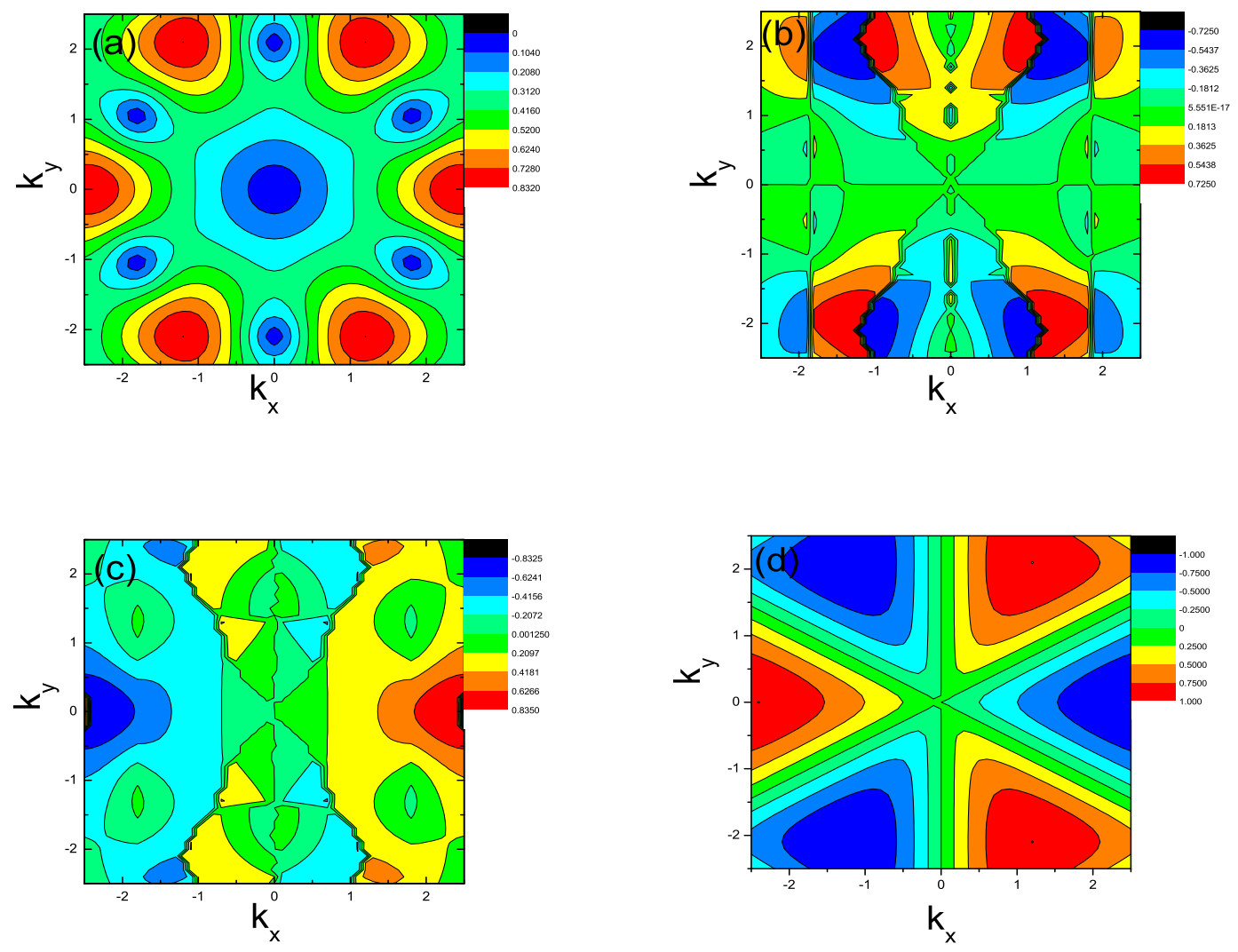

Figure 2: The symmetry of intraband pairing $\Delta_{11}$. In (a) the magnitude, (b) real part and (c) imaginary part of $\Delta_{11}$ for $\Omega / t_{\|}=1.0$ are shown. For comparison (d) plots $\Delta_{11}$ for $\Omega / t_{\|}=0$. 


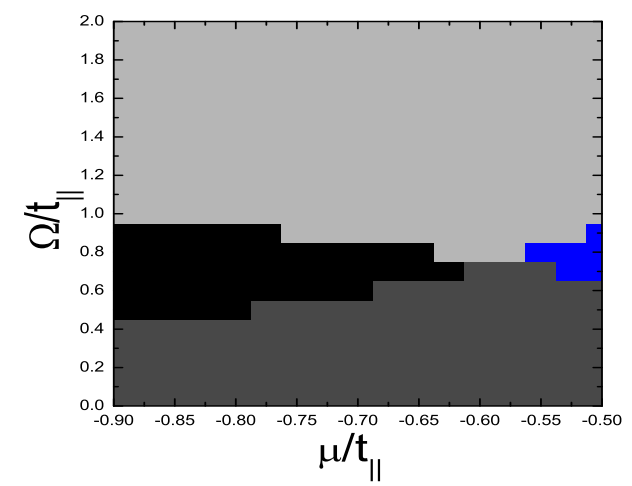

Figure 3: Topological phase diagram of the Hamiltonian (11) at the mean-field level. The light grey, dark grey, black and blue colors correspond to $I_{T K N N}=1,0,-1,2$ respectively. Parameter $U / t_{\|}=3.0$. 

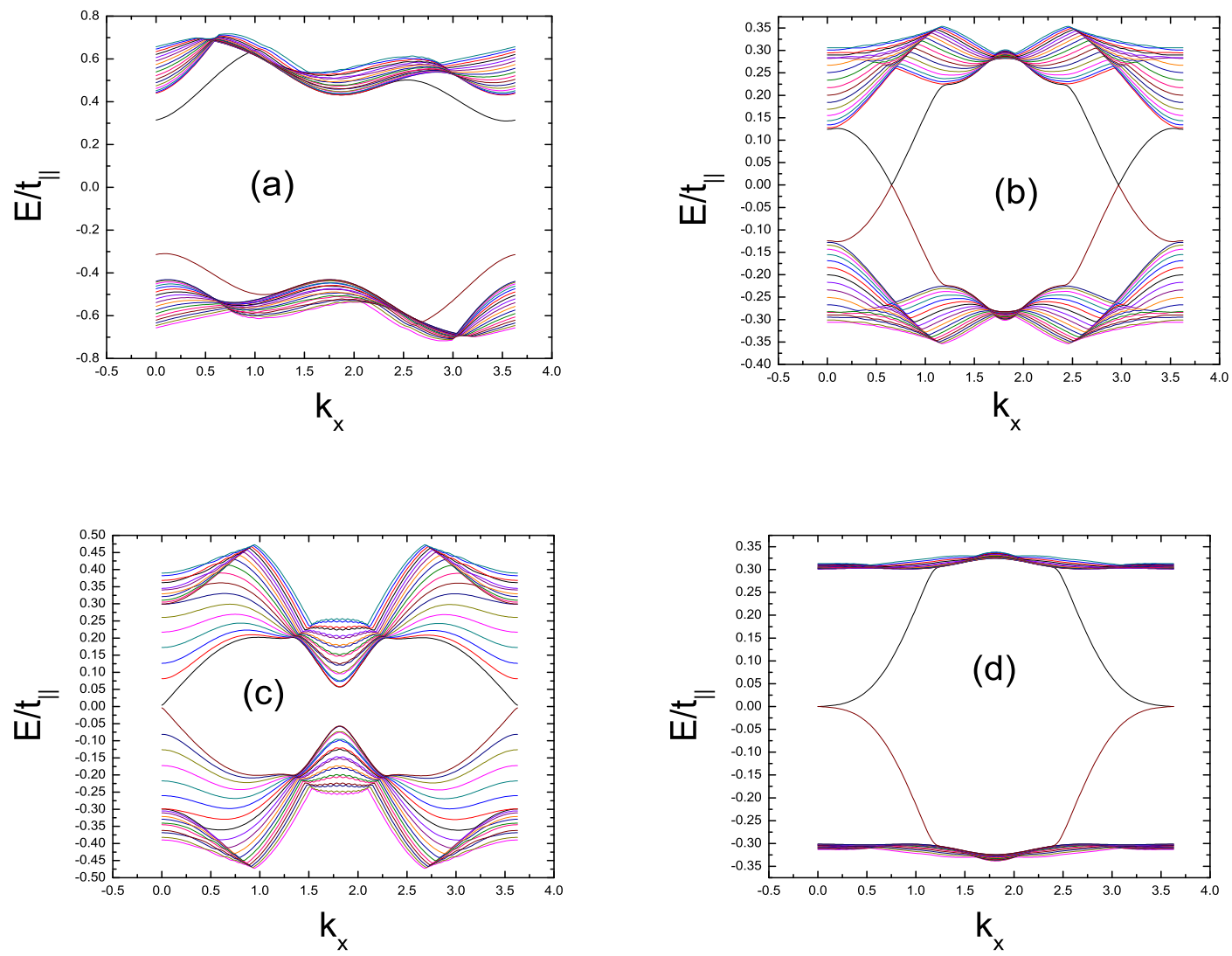

Figure 4: The gapless edge states with the open boundary condition along the zigzag edge of the honeycomb lattice. In (a) $I_{T K N N}=0, \mu / t_{\|}=-0.75, \Omega / t_{\|}=0.3, \Delta_{A} / t_{\|}=$ $0.969, \Delta_{B} / t_{\|}=0.063, \Delta_{C D W} / t_{\|}=1.462$, (b) $I_{T K N N}=2, \mu / t_{\|}=-0.5, \Omega / t_{\|}=0.8$, $\Delta_{A} / t_{\|}=\Delta_{B} / t_{\|}=0.288, \Delta_{C D W} / t_{\|}=0,(\mathrm{c}) I_{T K N N}=-1, \mu / t_{\|}=-0.85, \Omega / t_{\|}=0.8$, $\Delta_{A} / t_{\|}=\Delta_{B} / t_{\|}=0.523, \Delta_{C D W} / t_{\|}=0$, (d) $I_{T K N N}=1, \mu / t_{\|}=-0.65, \Omega / t_{\|}=1.1$, $\Delta_{A} / t_{\|}=\Delta_{B} / t_{\|}=0.307, \Delta_{C D W} / t_{\|}=0$. 